\title{
Giant Shocks in the Fermi Bubbles and the Origin of the Microwave Haze
}

\author{
Roland Crocker*广 \\ Research School of Astronomy and Astrophysics, Australian National University \\ E-mail: rcrockerefastmail.fm
}

\section{Geoffrey V. Bicknell}

Research School of Astronomy and Astrophysics, Australian National University

Andrew M. Taylor

Dublin Institute of Advanced Studies, Dublin, Ireland

\section{Ettore Carretti}

INAF - Osservatorio di Cagliari, Via della Scienza 5, 09047 Selargius, Italy

\begin{abstract}
Analysis of $\gamma$-ray data provided by the Fermi-LAT has revealed giant, hard-spectrum $\gamma$-ray lobes emanating from the Galactic nucleus (and extending to $\mathrm{lbl} \sim 50^{\circ}$ ). These 'Fermi Bubbles' have a hard-spectrum, total-intensity microwave ( $\sim 20-40 \mathrm{GHz})$ counterpart (the microwave Haze' extending to $\mathrm{lb} \sim 35^{\circ}$ ) and a steep spectrum, polarised radio $(2-20 \mathrm{GHz})$ counterpart (the 'S-PASS Lobes' extending to $|\mathrm{b}| \sim 60^{\circ}$ ). We present a unified model for these disparate, non-thermal phenomena in which all the structures are ultimately created by a wind driven by star formation in the central molecular zone of the Galactic Centre. Giant reverse shocks located $\sim$ kpc above and below the nucleus accelerate relativistic electrons, accounting for the microwave Haze. The $\gamma$-rays are mostly produced by hadronic emission through shock accelerated relativistic protons interacting with dense, thermally unstable clouds within the Bubbles but concentrated near their edges. The Bubbles are currently slowly expanding with an age of a few $\times 100 \mathrm{Myr}$.
\end{abstract}

The 34th International Cosmic Ray Conference,

30 July- 6 August, 2015

The Hague, The Netherlands

\footnotetext{
*Speaker.

$\dagger$ Australian Research Council Future Fellow.

${ }^{\ddagger}$ Schrödinger Fellow
} 


\section{Introduction}

This work is based on [6]. The Fermi Bubbles are giant $\gamma$-ray structures emanating north and south from the Galactic nucleus [11,21, 24, 1] and extending $\sim 8 \mathrm{kpc}$ above the Galactic plane.

Many models and ideas have been proposed to explain the Bubbles and both their ultimate energy source and the $\gamma$-ray emission process that illuminates them remain in contention. The Bubbles might be the signatures of a fairly recent activity of the central supermassive black hole $[21,25,18,15,23,14,22,20]$. In this case, inverse Compton (IC) radiation of cosmic ray electrons is a natural candidate to explain the $\gamma$-ray emission. This follows because the $\lesssim$ Myr loss timescale of the $\gtrsim \mathrm{TeV}$ electrons required to upscatter $\mathrm{CMB}$ photons to $\gtrsim \mathrm{GeV} \gamma$-ray energies implies that the Bubbles have to be expanding highly supersonically with their total energy content, $\sim 10^{56}$ erg, injected explosively. The black hole is quite capable of doing this. But a problem this idea encounters is that hot, shock heated gas radiating at X-ray energies at the Bubbles' edges is then expected. While some thermal X-ray emission coincident with the edges does seem to be present at lower latitudes [3], searches for such emission at higher latitudes do not show this gas gas [16].

An alternative class of models invokes Bubble inflation from the long-timescale integration of energy injected by more-or-less secular processes associated with the Galactic nucleus, e.g., tidal disruption events that occur there every $10^{4}-10^{5}$ year [5] or the concentrated and sustained starformation activity [17] that occurs throughout the nuclear region (inner 200-300 diameter region around the black hole). This sort of idea complements the possibility that the Bubbles' $\gamma$-rays originate not from IC emission but from hadronic collisions experienced by a population of cosmicray protons and heavier ions $[9,8,7]$. Such ions lose energy much more slowly than the high-energy electrons requisite for IC $\gamma$-ray production, thereby allowing the total current energy content of the Bubbles to be accumulated more gradually from relatively less powerful processes.

Features coincident with the Bubbles or some component of the Bubbles have been discovered in total intensity radio and microwave emission, polarized radio and microwave emission, and (as already mentioned) X-ray emission [13, 12, 2, 4, 3, 16]. Prima facie the existence of microwave emission coincident with the Bubbles - at least in their lower reaches - seems to constitute evidence in support of an IC scenario; the same electron population that radiates via IC into $\gamma$-ray energies can supply the observed microwave 'haze' via synchrotron radiation with the correct spectrum $[21,1]$. Furthermore, while hadronic models also predict some concomitant synchrotron emission from secondary electrons and positron created in $p p$ collisions, it is difficult to reconcile the flat spectrum of the observed haze with the expected steep spectrum of synchrotron radiation from a 'cooled' secondary electron population [1,5]. Moreover, it is already known [4] that the power in cosmic ray primary electrons accelerated in concert with nuclear star formation is a good match to the Bubbles' $2.3 \mathrm{GHz}$ synchrotron luminosity. It seems, then, that even a hadronic scenario requires primary electrons at some level to explain the totality of Giant Outflow phenomenology.

Relatedly, a point in support of the star-formation-driven hadronic model $[9,8,7]$ is that the power currently being delivered into cosmic ray ions by nuclear star-formation is elegantly sufficient to explain the Bubbles' (putatively hadronic) $\gamma$-ray luminosity in steady state.

Here we set out a revised star-formation-driven model for which the $\gamma$-ray emission from the Bubbles is still dominantly hadronic but also contains a non-negligible IC component at lower latitudes. The microwave Haze is supplied by primary electron synchrotron. The primary electron 
population required to supply these radiations is (re)accelerated at giant shocks at $\sim$ kpc heights into each Bubble as we now explain. We also show that the Bubbles' contact discontinuity edges are currently expanding slowly into the Galactic halo and that the Bubbles have a characteristic age of $\sim$ few $\times 10^{8} \mathrm{yr}$, similar to the cooling time of the X-ray emitting plasma inside them.

\subsection{Outline of model}

The main features of our model for the Bubbles' structure and non-thermal emission phenomenology are shown in Figure 1 and described below; more details are in [6].

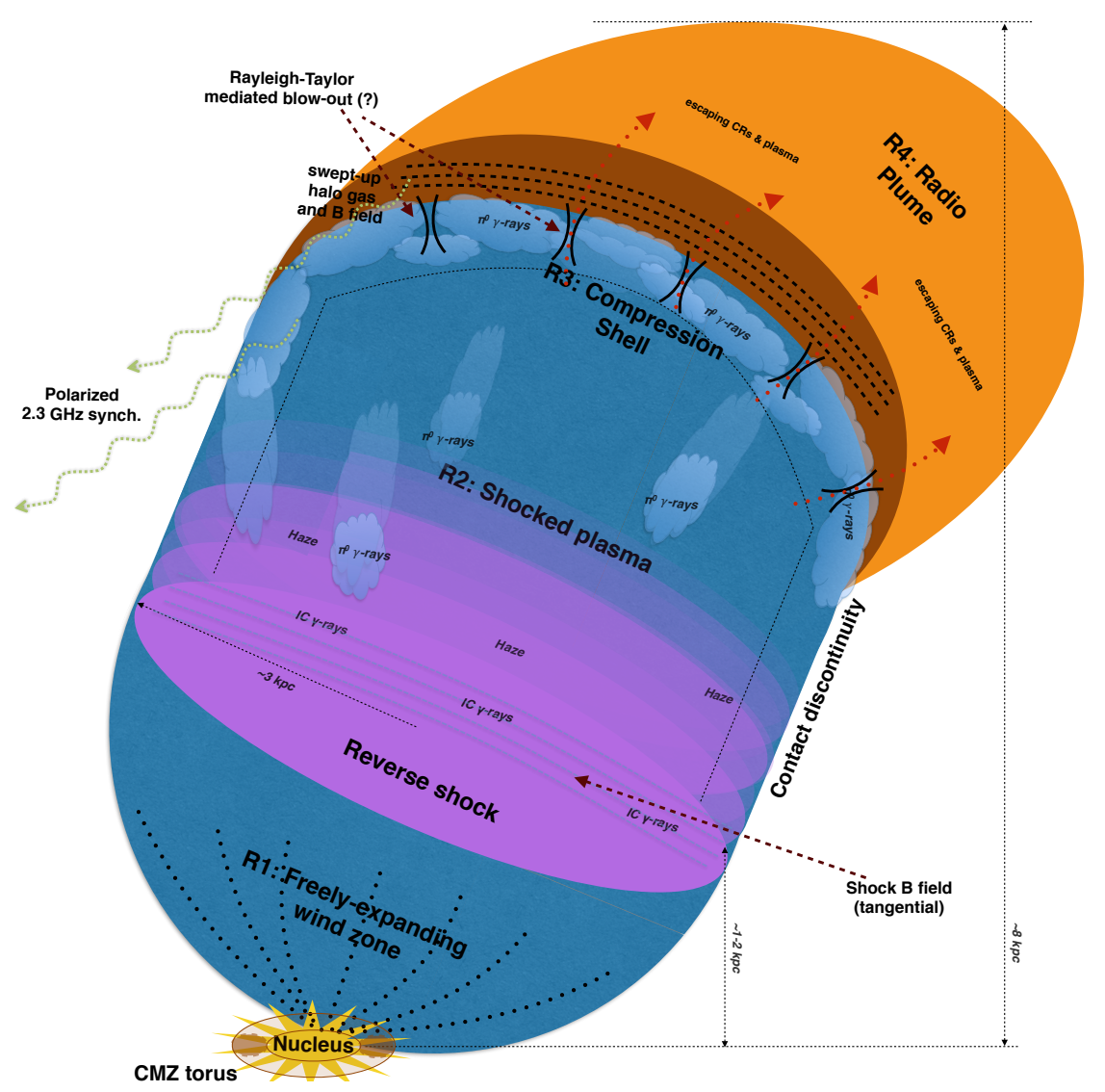

Figure 1: Schematic showing the main features of bubble model reproduced from [6]

1. Co-entrained hot plasma, magnetic fields, and cosmic rays are energised within the central $\sim 100 \mathrm{pc}$ (radius) region of the Galactic nucleus. The pressure gradient supplied by these ISM phases accelerates a wind that quickly reaches $\gtrsim 500 \mathrm{~km} / \mathrm{s}$. The massive $H_{2}$ torus circumscribing the injection zone collimates the wind, directing it perpendicular to the plane.

2. The Bubbles are inflated by the injection of hot plasma in the nuclear outflow over timescales $\gtrsim$ few $\times 10^{8}$ year. The mean mechanical power and mass flux into the bases of the Bubbles are $\sim 10^{40} \mathrm{erg} / \mathrm{s}$ and $\sim 0.1 M_{\odot} /$ year (the latter similar to the star formation rate [8]). 
3. This outflow, however, is not powerful enough - given the strong gravitational field, the pressure of the overlying halo gas, and the occurrence of gas cooling - to break out of the Galaxy. Note that recent work indicates the gas density and pressure in the halo, especially in the vicinity of the Bubbles, is larger than has often been assumed in the past [19].

4. The Bubbles develop the classical wind-blown bubble structure of (starting from the smallest scales): i) a reverse shock, ii) a contact discontinuity (CD), and a forward shock.

5. The CD separates ISM material swept-up and shocked in the downstream of the forward shock from shocked Bubble material in the downstream of the internal reverse shock. (In our model, the $\gamma$-ray edge of the Bubbles is identified with the contact discontinuity.)

6. The Bubbles quickly (within $\sim 10^{7}$ year) reach pressure equilibrium with their environment; at this point the forward shock degenerates into a sound wave. The CD decelerates even faster and is subsonic after only $\lesssim 10^{6}$ year; at present it is expanding at only $\sim$ few $\mathrm{km} / \mathrm{s}$.

7. Thermal plasma and cosmic rays initially suffer adiabatic energy losses as they are blown out of the nucleus on the expanding outflow (R1 in fig 1). However, they encounter giant reverse shocks where the flow ram pressure equilibrates with the thermal pressure of the shocked, downstream gas (cf. [17]). Here the plasma and cosmic-rays are re-heated/re-accelerated.

8. There is energy-independent transport of the non-thermal particles into the Bubbles mediated by the $\sim 100-150 \mathrm{~km} / \mathrm{s}$ sub-sonic post-shock flow in combination with cosmic-ray streaming. High-energy cosmic-ray electrons produce inverse-Compton and synchrotron emission on photon and magnetic fields in the shock-downstream. Given there is a systematic flow, the spectrum of these electrons retains the hard injection distribution up to some distancedependent maximal energy above which the spectrum is exponentially suppressed. Given the slow transport speeds and short cooling times, microwave-emitting electrons reach only $\sim$ $1 \mathrm{kpc}$ past the shocks and $\gtrsim \mathrm{GeV}$ inverse-Compton-emitting electrons only reach few $\times 100$ pc. In contrast, cosmic-ray protons and 2.3-GHz-synchrotron-emitting electrons survive to reach the full extent of the Bubbles. The hard spectrum synchrotron radiation from cosmicray electrons reaccelerated on the shocks and transported downstream provides an excellent match to the luminosity, spectrum, distribution, and morphology of the microwave Haze [6].

9. As the Bubbles age, the radiative losses of the plasma over their entire volume gradually become more significant. An increasingly large fraction of the hot plasma mass injected into the Bubbles is lost by drop-out as cooling leads to local thermal instability that causes individual parcels of gas to cool, collapse, lose buoyancy, and fall out of the Bubbles.

10. Because of the density-squared dependence of cooling, this mass drop-out process dominantly occurs in the somewhat over-dense shell of material that forms inside of the CD where the slow flow of plasma away from the reverse shock is gradually arrested (R3 in Figure 1).

11. While the plasma evolves radiatively in the shell, cosmic ray hadrons and magnetic fields, in constrast, continue to evolve adiabatically. Their compression in the shell material leads to adiabatic energy gain; they are compressed until they equilibrate with the thermal pressure of the volume-filling plasma in R2 between the shell and the reverse shock [7]. 
12. The ultimate extent and mass of the shell $\mathrm{R} 3$ is determined by pressure balance between Bubbles and halo atmosphere and the further condition that the shell's cooling radiation saturates the enthalpy flux represented by the slow flow of hot plasma into it from R2.

13. Both the mass of shell gas and the hadronic cosmic-ray energy density in the shell can be determined from the considerations given above, allowing us to calculate the hadronic emission from the shell; this saturates the observed $\gamma$-ray luminosity for reasonable parameters.

14. As for the ions, the lower-energy cosmic-ray electrons that live long enough to reach the shell are also adiabatically compressed and reenergised. Unlike the immediate shock downstream, in the shells there is a mixture of electrons with different ages and different characteristic energies. This leads to the integrated, steady-state shell electron spectrum being steepened by the canonical difference in spectral index of 1 associated with synchrotron/IC losses. This cooled primary electrons synchrotron-radiate on the adiabatically-compressed shell magnetic field, explaining the steep-spectrum, polarized emission measured between 2.3 and $23 \mathrm{GHz}$.

15. The shell/CD does not constitute a perfect boundary, however: at $2.3 \mathrm{GHz}$ polarisation there are plumes of radio emission that extend north-west and south-west beyond the Bubbles' $\gamma$-ray boundaries (R4). We speculate that these signify the break-out of the light fluid of co-mixed cosmic rays, plasma, and magnetic fields through the heavy shell, mediated by the Rayleigh-Taylor instability. However, the CD generates a $\gamma$-ray edge because, even if some cosmic-ray protons escape through it, it delimits the shell of dense target gas.

\section{Presence of giant reverse shocks in both Bubbles}

The location of each Bubble's reverse shock is determined from the condition that the ram pressure of the wind and the internal bubble pressure, $p_{b}$ are related by the Rankine-Hugoniot condition. For a conically expanding outflow with solid angle $\Omega_{w}$, the shocks are located at

$$
R_{\text {shock }}=\left(\frac{2}{\gamma+1} \frac{\dot{M} v_{\mathrm{w}}}{p_{\mathrm{b}} \Omega}\right)^{1 / 2} \simeq\left(\frac{2}{\gamma+1}\right)^{1 / 2} \frac{\left(2 \dot{E}_{0} \dot{M}\right)^{1 / 4}}{\left(p_{\mathrm{b}} \Omega\right)^{1 / 2}}\left(1-\frac{\dot{M}}{\dot{E}_{0}} \Delta \phi\right)^{1 / 4},
$$

where $\Delta \phi$ is the difference in gravitational potential between the nuclear injection zone and at the shock. This equation has to be solved numerically for a particular choice of the gravitational potential; we find $R_{\text {shock }} \sim \mathrm{kpc}$ for fiducial parameters [6]. Notable is that for the comparatively puny Galactic Centre wind gravitational losses in the outflow cannot be ignored and the wind speed is significantly slowed, from $\sim 700$ to $\sim 500 \mathrm{~km} / \mathrm{s}$, before the shocks are reached.

\section{Slow expansion of Bubbles' edges}

Semi-analytical equations governing the expansion of a radiative bubble expanding into an atmosphere of finite pressure are described in [6]. The results of this calculation for fiducial parameters are shown in fig. 2; notable is that the Bubbles' edges are presently expanding highly sub-sonically at a speed of only $\sim$ few $\mathrm{km} / \mathrm{s}$. Important in informing this slow expansion are: the lower mechanical power of the outflow; the existence of non-negligible gravitational losses; the existence of non-negligible cooling losses; and the existence of non-negligible atmospheric pressure. 


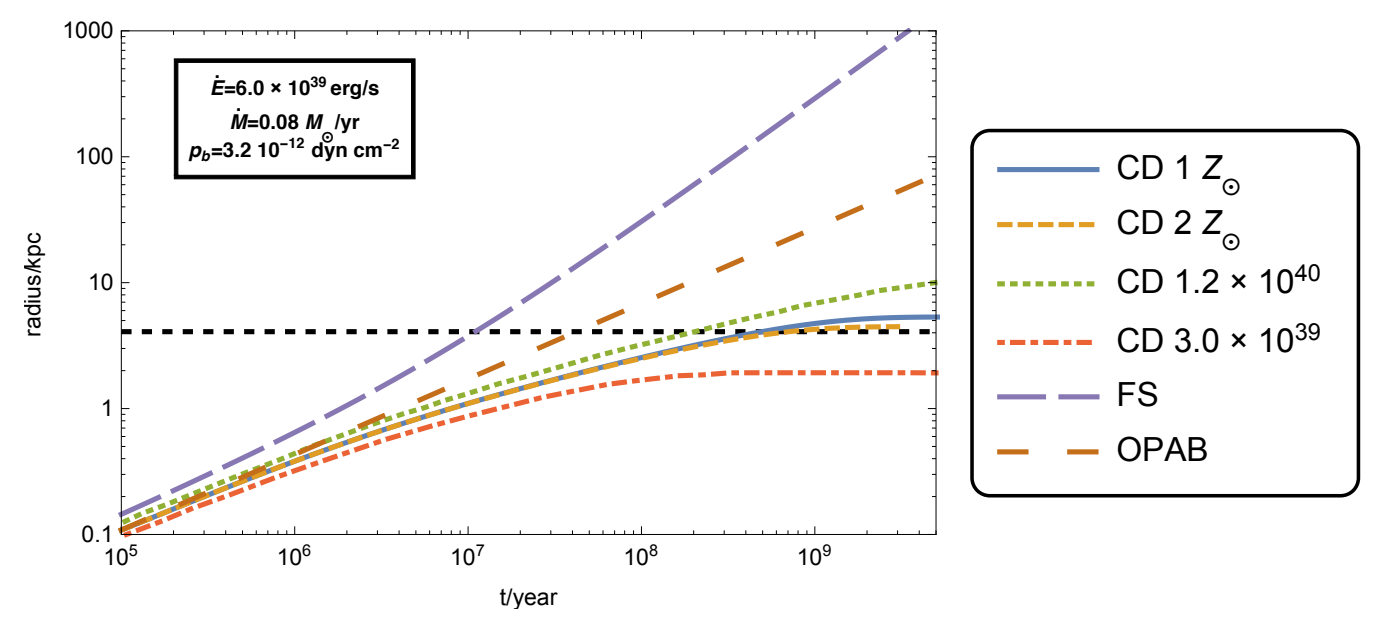

Figure 2: Radius of the bubble contact discontinuity (CD) and forward shock (FS) vs. time for $\dot{E}=$ $6 \times 10^{39} \mathrm{erg} / \mathrm{s}$ and solar metallicity (unless otherwise marked) and $\dot{M}=0.08 M_{\odot} / \mathrm{yr}$; the atmosphere has pressure $2.0 \mathrm{eV} \mathrm{cm}^{-3} \simeq 3.2 \times 10^{-12} \mathrm{dyn} \mathrm{cm}^{-2}$. CD curves are: i) solid blue for $\dot{E}=6.0 \times 10^{39} \mathrm{erg} / \mathrm{s}$; ii) dashed orange for $\dot{E}=6.0 \times 10^{39} \mathrm{erg} / \mathrm{s}$ and cooling with twice solar metallicity $\left(Z=2 Z_{\odot}\right)$; iii) dotted green for $\dot{E}=1.2 \times 10^{40} \mathrm{erg} / \mathrm{s}$; and iv) dot-dashed orange for $\dot{E}=3.0 \times 10^{39} \mathrm{erg} / \mathrm{s}$. The long dashed violet curve is for the forward shock. The brown long-dashed curve shows the analytical result for the expansion of an over-pressured, adiabatic bubble (OPAB); this very substantially over-estimates the bubble radius for $t \gtrsim$ few $\times 10^{7} \mathrm{yr}$. The horizontal black dashed line shows the equivalent spherical radius of $4.1 \mathrm{kpc}$ for the total volume of the Fermi Bubbles; the fiducial model bubble has attained this size by $\sim 5 \times 10^{8} \mathrm{yr}$.

\section{4. $\gamma$-ray emission from the Bubbles}

A successful model of the Bubbles' $\gamma$-ray phenomenology needs to explain their luminosity, spectrum, and morphology. Generic to any hadronic model is a $\sim \mathrm{GeV}$-scale low energy down-turn feature in the spectral energy distribution; this is broadly consistent with the spectral phenomenology [9]. We show in below that our model provides a good match to the overall $\gamma$-ray luminosity of the Bubbles. In addition, for acceptable regions of the $\left\{\dot{E}_{0}, \dot{M}\right\}$ parameter space (mechanical energy and mass injection rates into the outflows) we find [6] moderately high Mach numbers (6-9) at the shocks, naturally accounting for the hard 1-100 GeV spectrum of the Bubbles and the hard microwave Haze. We also show in [6] that our model predicts the volumetric emission from the Bubbles should peak towards their edges, consistent with their flat (projected) surface brightness.

Adapting the calculations presented by [7], we can calculate the hadronic luminosity of the Bubbles. The Bubbles' radiative plasma collapses into cooling condensations; these form preferentially in the shell region (R3 in fig. 1) leading up to the contact discontinuity. Magnetic fields and cosmic rays, gathered into the condensations, however, evolve adiabatically. In fact, compression of the shell by the plasma in R2 is arrested when the adiabatic energy-gain of magnetic fields and cosmic rays results in their reaching pressure equilibrium with the interior plasma. The size of the shell is dually controlled by this together with the requirement that the cooling radiation taking place within it (strictly, in the steady state limit) balances the enthalpy flux represented by the flow of hot plasma from the hot interior. Thus adiabatically compressed cosmic rays are concentrated together with the relatively dense, cool gas in the condensations. This co-location of these com- 
ponents is the essential ingredient to allow a hadronic model to work on the same few $\times 10^{8} \mathrm{yr}$ timescale as the expansion of the Bubbles despite the low initial density of the interior plasma. We are ultimately able to estimate the $1-100 \mathrm{GeV}$ luminosity of the Bubbles as

$$
\begin{aligned}
L_{\gamma}^{\mathrm{pp}} & \simeq \frac{15 f_{\mathrm{bol}}}{8} \frac{\left(k_{B} T_{\mathrm{FB}}\right)^{2} \dot{M}}{\mu m \Lambda\left[T_{\mathrm{shell}}\right]} \frac{n_{H}}{n_{\mathrm{tot}}}\left(\frac{L_{\mathrm{CR}}^{\mathrm{FB}}}{\dot{E}_{\mathrm{therm}}^{\mathrm{FB}}}\right)^{3 / 4} \sigma_{\mathrm{pp}} \kappa_{\mathrm{pp}} c \\
& \simeq 2.4 \times 10^{37} \mathrm{erg} / \mathrm{s}\left(\frac{T_{\mathrm{FB}}}{6.0 \times 10^{6} \mathrm{~K}}\right)^{2}\left(\frac{\dot{M}}{0.1 M_{\odot} / \mathrm{yr}}\right)\left(\frac{L_{\mathrm{CR}}^{\mathrm{FB}}}{\dot{E}_{\mathrm{therm}}^{\mathrm{FB}}} / 0.1\right)^{3 / 4}
\end{aligned}
$$

which compares favourably to the $\sim 2 \times 10^{37} \mathrm{erg} / \mathrm{s} 1-100 \mathrm{GeV}$ luminosity of the Bubbles if $\gtrsim 10 \%$ of the mechanical power at the shock is delivered to cosmic rays. Interestingly, IC emission from primary electrons (re)-accelerated at the giant shocks makes a sub-dominant but non-negligible ( $\sim 10 \%)$ contribution to the Bubbles' overall $\gamma$-ray luminosity but this emission does not extend more than $\sim$ few $\times 100$ pc past the shocks given the electrons' short loss times.

\section{Synchrotron emission from the Bubbles}

We explain briefly how our model accounts for the complicated synchrotron emission phenomenology of the GC's giant outflows: The nucleus directly accelerates a population of hardspectrum primary electrons that is advected from the region before it can lose much energy radiatively in situ $[10,8,4]$. These electrons cool adiabatically and radiatively in the expanding outflow (R1) until they encounter the giant reverse shock in either bubble where they are reaccelerated. At latitudes higher than the shocks, there are then three zones for synchrotron emission:

1. A zone downstream of each reverse shock, partially encompassing R2, where the electrons are conveyed downstream by the downstream flow plus their streaming along magnetic field lines. In this zone, there is a 1-to-1 correspondence between distance from the shock and electron 'age'. The frequency-dependent extent of this region is given approximately by the requirement that the transport time from the shock be less than the total cosmic ray electron loss time. The synchrotron spectrum within this zone is thus 'uncooled', $F_{v} \sim v^{-0.5}$, reflecting that the electron spectrum is simply the hard injection spectrum produced at the shock, $d N_{e} / d E_{e} \sim E_{e}^{-2}$ up to some maximum energy above which it is exponentially cut-off. The hard-spectrum microwave Haze is largely attributable to the synchrotron radiation from these electrons radiating on the magnetic field in the base of $\mathrm{R} 2$.

2. The compression shell that covers the entire solid angle of the $\gamma$-ray Bubbles out to the contact discontinuity, R3. Here the adiabatically amplified magnetic field is rather strong and regular. Synchrotron emission here is by i) the adiabatically-compressed, lower-energy and longer lived primary electrons that can travel all the way from the shock and ii) secondary electrons from $p p$ collisions in the shell gas. Note that i) given relative transport and loss times microwave-emitting primary electrons accelerated at the shock never reach $R 3$ and ii) in R3, there is a mixture of electrons of different ages as shell electrons simply cool in situ. The latter implies that, above some break energy the integrated, steady-state shell electron spectrum of R3 is $d N_{e} / d E_{e} \sim E_{e}^{-3}$ implying a synchrotron spectrum above $v_{\mathrm{bk}}$ of $F_{v} \sim v^{-1}$. This explains the radio lobes' steep and polarized spectrum between 2.3 and $23 \mathrm{GHz}$. 
3. A zone identified with the polarized radio plumes that extend beyond the boundaries of the north and south bubbles at high latitudes.

In summary, detailed calculations [6] show that our model reproduces the spectrum, morphology, and luminosity of the hard spectrum microwave haze and the steep spectrum, polarized radio lobes. To our knowledge, our model is the first to have achieved a simultaneous explanation of the $\gamma$-ray, radio continuum, and microwave emission from the Galactic Centre's giant outflows.

\section{References}

[1] Ackermann et al. (Fermi-LAT Collaboration) 2014, arXiv:1407.7905

[2] Ade, P. A. R., Aghanim, N., et al. (Planck Collaboration) 2013, A\&A , 554, A139

[3] Bland-Hawthorn, J., \& Cohen, M. 2003 ApJ, 582, 246

[4] Carretti, E. et al. 2013, Nature , 493, 66

[5] Cheng, K. S., Chernyshov, D. O., Dogiel, V. A., \& Ko, C. M. 2014, arXiv:1411.6395v1

[6] Crocker, R. M., Bicknell, G. V., Taylor, A. M., \& Carretti, E. 2014, arXiv:1412.7510, in press ApJ

[7] Crocker, R. M., Bicknell, G. V., Carretti, E., Hill, A. S., \& Sutherland, R. S. 2014, ApJ, 791, L20

[8] Crocker, R. M. 2012, MNRAS, 423, 3512

[9] Crocker, R. M., \& Aharonian, F. 2011, Phys. Rev. Lett., 106, 101102

[10] Crocker, R. M. et al., 2011b, MNRAS, 413, 763

[11] Dobler, G. et al. 2010, ApJ, 717, 825

[12] Dobler, G. 2012, ApJ, 750, 17

[13] Finkbeiner, D. P. 2004, ApJ, 614, 186

[14] Fujita, Y., Ohira, Y., \& Yamazaki, R. 2013, ApJ, 775, L20

[15] Guo, F., \& Mathews, W. G. 2012, ApJ, 756, 181

[16] Kataoka, J., Tahara, M., Totani, T., et al. 2013, ApJ, 779, 57

[17] Lacki, B. C. 2014, MNRAS, 444, L39

[18] Mertsch, P., \& Sarkar, S. 2011, Phys. Rev. Lett.,, 107, 091101;

[19] Miller, M. J., \& Bregman, J. N. 2014, arXiv:1412.3116

[20] Mou, G., Yuan, F., Bu, D., Sun, M., \& Su, M. 2014, ApJ, 790, 109

[21] Su, M., Slatyer, T. R., \& Finkbeiner, D. P. 2010, ApJ, 724, 1044

[22] Wardle, M., \& Yusef-Zadeh, F. 2014, ApJ, 787, LL14

[23] Yang, H.-Y. K. et al., Ruszkowski, M., Ricker, P. M., Zweibel, E., \& Lee, D. 2012, ApJ, 761, 185;

[24] Yang, R.-z., Aharonian, F., \& Crocker, R. 2014, A\&A , 567, A19

[25] Zubovas, K., King, A. R., \& Nayakshin, S. 2011, MNRAS, 415, L21-L25 